\title{
Concurrency Relations between Digital Planes
}

\author{
Peter Veelaert, Maarten Slembrouck, and Dirk Van Haerenborgh \\ Ghent University, \\ Valentin Vaerwyckweg, B9000 Ghent, Belgium \\ \{peter. veelaert, maarten. slembrouck, dirk. vanhaerenborgh\}@ugent. be
}

\begin{abstract}
In this paper we examine concurrency relations between planes whose position is not precisely known. The simplest case consists of four planes, where we have to determine whether the four planes can be forced to pass through one common intersection point by moving them slightly within specified limits. We prove that if such a concurrency relation is possible then it can be found in a finite number of steps by a simple geometrical construction. This result remains valid for larger collections of planes, with multiple concurrency relations, provided each pair of relations shares at most one plane, and the relations do not form cycles.
\end{abstract}

\section{Introduction}

In $3 \mathrm{D}$ concurrencies of planes occur when at least 4 planes meet at a common point. However, when the planes are extracted from real data, e.g., point clouds, even when four planes are concurrent in the real scene, the extracted planes will not meet at a common point, because of imprecise data. Concurrencies may still be possible, but within prespecified tolerances for the positions and slopes of the planes. Our goal is to find hidden concurrencies by simple geometric constructions that are easy to implement. A geometric construction here means that the concurrent planes pass through lines and points constructed from intersections and spans of a finite set of initial points. When the initial points have integral coordinates all the computations can be done with rational numbers, with the significant advantage that the results are exact.

In this paper the tolerances on the position of a plane are defined by a convex polytope, which we will call a domain. Thus given a set of $n$ domains and a set of $m$ concurrency relations, our goal is to determine whether the concurrency relations can hold for $n$ plane positions chosen from the domains.

Part of this work is motivated by the renewed interest in visual hulls [1]. Although known for some time [2, 3], multi-camera systems now provide means to acquire detailed visual hulls in real time. One former obstacle for acquiring visual hulls was that they need accurate silhouettes. Although obtaining reliable silhouettes from image segmentation remains difficult [4], motion detection algorithms with sophisticated background models are now able to extract silhouettes

that are sufficiently reliable [5, 6]. Each camera added to the network improves the accuracy of the hull. In fact, an abundance of cameras allows us to deal with occlusions and missing segments.

R. Gonzalez-Diaz, M.-J. Jimenez, B. Medrano (Eds.): DGCI 2013, LNCS 7749, pp. 347-359, 2013.

(C) Springer-Verlag Berlin Heidelberg 2013 
Fig. 11 shows a synthetic image of the visual hull of a cubic box when viewed by 4 cameras. The visual hull is a polytope which results from the intersection of 4 cones, and the halfspace defined by the ground plane. Each cone is generated by the light rays that pass through the projection center of one of the cameras and the silhouette of the box as seen by this camera. The precise combinatorial structure of the visual hull depends on the position of the box relative to the cameras. Thus the number of vertices, edges, and facets may vary when a box is displaced. However, at a corner of the box, typically the visual hull will have multiple supporting planes that meet at a common point. For example, in Fig. 1 , six planes meet at the corner in front. Clearly, if we want to embed a box inside a visual hull, it is interesting to know where $n$ planes, with $n>3$, meet at a common point. Fig. 2 shows the real data for a rectangular box. Because of noisy data, concurrencies that should occur at corners are not present, but slight displacements of the facets can make them reappear.

(a)

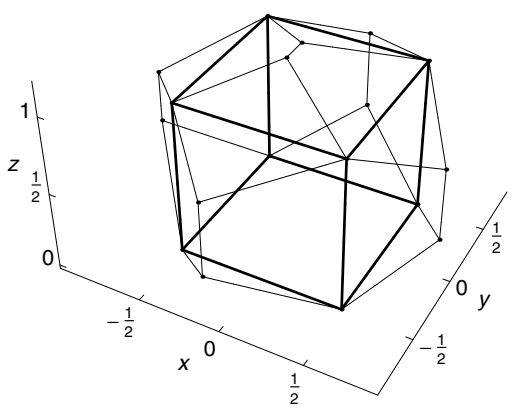

(b)

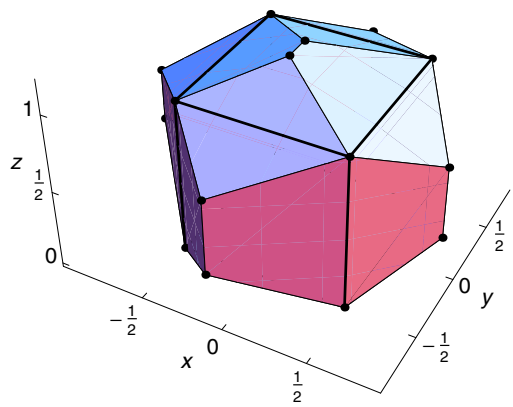

Fig. 1. Synthetic generation of a visual hull. (a) shows the edges of the box (bold lines) together with the edges of its visual hull. (b) shows the same result but with facets shown as filled polygons. Because of the edges and facets are quite small, a slight change of the camera positions may already give a different combinatorial structure.

Tolerances for planes and lines also arise naturally in digital geometry 7 9]. The results proven in this paper closely resemble previous results on the concurrency of lines [10]. As before, the domain of a plane [11], i.e., the set of all parameters that fall within the prespecified tolerances, plays an important role, since all proofs are given in the dual parameter space.

Section 2 introduces concurrency relations in the primal as well as in the dual space. In Section 3 we clarify what it means for a concurrency relation to be constructible. The paper is concluded in Section 4. 
(a)

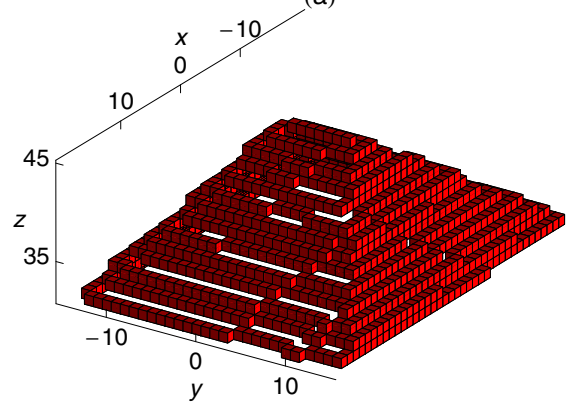

(b)

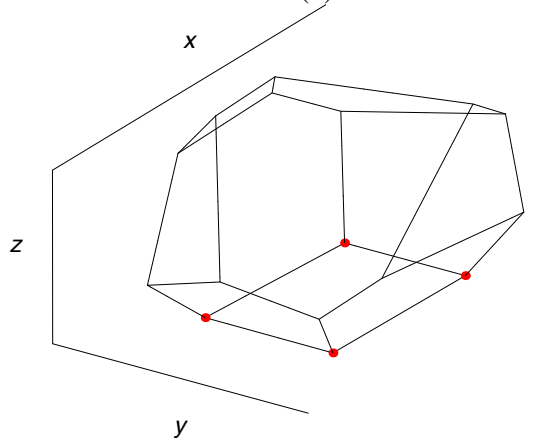

Fig. 2. Visual hull of a box acquired by 4 cameras. (a) Shows a voxel representation of a close up of the upper part of the visual hull. Because each horizontal layer of the visual hull is constructed separately, the surface contains gaps between voxels. (b) Shows the result of segmentation, and then fitting planes to each segment. The dots indicate the vertices that rest on the ground plane. The combinatorial structure depends strongly on the fitting process. For example, $L_{\infty}$ fitting gives a different outcome than $L_{2}$ fitting.

\section{Concurrency Relations}

\subsection{Concurrencies in Primal Space}

A convenient way to model the tolerance of a plane is to describe its parameters by a convex polytope, also called the domain. There are several ways to arrive to such a domain. Let $W$ be a finite set of points in $\mathbb{R}^{3}$, and let $\tau>0$ be a positive thickness. Then the set of all positions the plane can take such that it passes within a distance $\tau / 2$ of $W$ is defined as the set of parameter points $(a, b, c)$ that satisfy

$$
-\frac{\tau}{2} \leq z_{i}-a x_{i}-b y_{i}-c \leq \frac{\tau}{2}, \quad \forall\left(x_{i}, y_{i}, z_{i}\right) \in W .
$$

The thickness $\tau$ can be chosen as large as needed to accommodate for the uncertainty of the point positions. Alternatively, the tolerance of a plane can be defined by the way it separates point sets. Let $U$ and $V$ be finite and non-empty subsets of $\mathbb{Z}^{3}$ such that $U$ can be linearly separated from $V$. Then each parameter $(a, b, c)$ point that satisfies

$$
\begin{array}{ll}
z_{i}-a x_{i}-b y_{i}-c>0, & \forall\left(x_{i}, y_{i}, z_{i}\right) \in U \\
z_{j}-a x_{j}-b y_{j}-c<0, & \forall\left(x_{j}, y_{j}, z_{j}\right) \in V .
\end{array}
$$

corresponds to a plane that separates the two subsets. We will define the domain $D(U, V)$ as the set of parameter points $(a, b, c)$ that satisfy

$$
\begin{array}{ll}
z_{i}-a x_{i}-b y_{i}-c \geq 0, & \forall\left(x_{i}, y_{i}, z_{i}\right) \in U \\
z_{j}-a x_{j}-b y_{j}-c \leq 0, & \forall\left(x_{j}, y_{j}, z_{j}\right) \in V .
\end{array}
$$


Thus $D(U, V)$ is the topological closure of the set defined by (11). In general a domain is a convex polyhedron. If the inequalities are such that the domain is bounded, it is a polytope. A domain becomes infinite if the position of one of the separating planes is orthogonal to the $x y$-plane. To avoid this complication, we will assume that there exists at least one plane in space to which none of the separating planes are orthogonal. Then, we can always rotate the coordinate axes such that all domains will be bounded convex polytopes, and all planes can be defined by an equation of the form $z-a x-b y-c=0$.

We will allow special kinds of domains. A domain will be a planar segment (or polygon) in $\mathbb{R}^{3}$ if we require that the plane that separates two point sets also has to pass through a given point $p_{1}$. Similarly, a domain will be a line segment if the separating plane has to pass through two points, and trivially, a domain is a single point if we require that the separating plane has to pass through three given points. These special cases will be useful when we want to verify some results in extreme situations.

Each domain $D$ defines a set of possible plane positions defined by the equation $z=a x+b y+c$, where $(a, b, c) \in D$. To define concurrencies, the plane at position $(a, b, c)$ will be denoted as $\Pi_{(a, b, c)}$. If we denote with $P_{\text {conc }}\left(a_{1}, \ldots, c_{4}\right)$ the determinant

$$
\left|\begin{array}{llll}
1 & a_{1} & b_{1} & c_{1} \\
1 & a_{2} & b_{2} & c_{2} \\
1 & a_{3} & b_{3} & c_{3} \\
1 & a_{4} & b_{4} & c_{4}
\end{array}\right|
$$

then the four planes $\Pi_{\left(a_{1}, b_{1}, c_{1}\right)}, \ldots, \Pi_{\left(a_{4}, b_{4}, c_{4}\right)}$ are concurrent if $P_{\text {conc }}\left(a_{1}, \ldots, c_{4}\right)=0$.

This polynomial will also be denoted as $P_{\text {conc }}\left(\Pi_{1}, \Pi_{2}, \Pi_{3}, \Pi_{4}\right)$.

Our main problem is now the following. Suppose we are given $n$ domains $D_{i}$ and $m$ geometric relations $P_{j}$. Can we find a plane configuration $\Pi_{\left(a_{1}, b_{1}, c_{1}\right)}, \ldots$, $\Pi_{\left(a_{n}, b_{n}, c_{n}\right)}$, with $\left(a_{i}, b_{i}, c_{i}\right) \in D_{i}$ such that $P_{j}\left(a_{i}, \ldots, b_{k}\right)=0$ holds for all $m$ relations $P_{j}$ ?

This is a non-linear programming problem with linear inequalities for the domains and non-linear equations for the concurrency relations. Hence, we may expect that in general a solution can only be found by solving systems of nonlinear equations. We shall show, however, that for certain configurations a solution can be found by a geometric construction. The feasibility of a geometric solution depends on how the concurrencies are linked to each other. An example of the configurations we envision is shown in Fig. 3. as a graphical diagram. Each node represents a plane, each quadrangle represents a concurrency relation. Thus Fig. 3 represents the concurrency relations $P_{\text {conc }}\left(\Pi_{11}, \Pi_{12}, \Pi_{13}, \Pi_{14}\right)$ and $P_{\text {conc }}\left(\Pi_{14}, \Pi_{22}, \Pi_{23}, \Pi_{24}\right)$, sharing one plane, $\Pi_{14}$.

The results we prove are valid for all chains of this type. Chains can be of arbitrary length, but two subsequent relations cannot share not more than one plane. Fig. 4 shows a chain with 3 relations. Obviously, we can also have multiple chains with no planes in common between chains. In this case each chain is handled separately. 


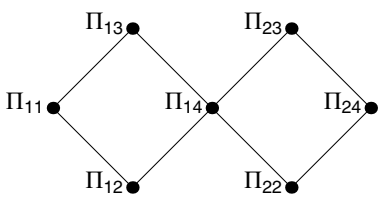

(a)

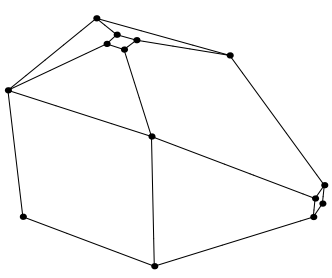

(b)

Fig. 3. (a) shows a chain with two concurrency relations, $P_{\text {conc }}\left(\Pi_{11}, \Pi_{12}, \Pi_{13}, \Pi_{14}\right)$ and $P_{\text {conc }}\left(\Pi_{14}, \Pi_{22}, \Pi_{23}, \Pi_{24}\right)$ share one plane $\Pi_{14}$. (b) shows how such a chain can arise in the primal space. A polytope has 2 small facets, each adjacent to 4 large facets (some facets are hidden). By changing the position of the large facets slightly, the small facets could disappear and be replaced by two vertices. Each of the five large facets in (b) corresponds to a plane $\Pi_{i j}$ in (a). The plane $\Pi_{14}$ corresponds to the large facet incident to both small facets.

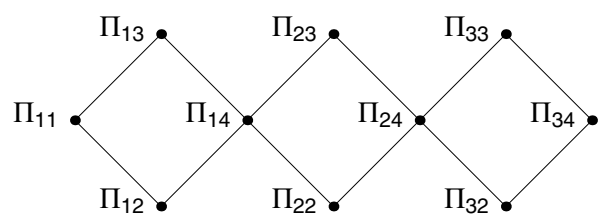

Fig. 4. A chain for three concurrency relations. A chain with $m$ relations will comprise $3 m+1$ planes. Planes are labeled such that plane $\Pi_{i j}$ is involved in the $i$-th relation. Planes that are involved in two relations receive labels of the form $\Pi_{i 4}$.

\subsection{Concurrencies in Dual Space}

We will call the $x y z$-space the primal space, and the parameter space the dual space. Geometrically, $n$ planes $\Pi_{\left(a_{i}, b_{i}, c_{i}\right)}$ in the primal space are concurrent if and only if their parameters $\left(a_{i}, b_{i}, c_{i}\right)$ are coplanar in the dual space. As a result, $n$ parameter domains will give rise to $n$ concurrent planes if we can find $n$ coplanar parameter points, one point from each domain. Clearly, this is equivalent to the assertion that there is at least one plane in the parameter space that crosses all the domains. Such a plane is called a common stabbing. Thus, finding concurrencies in the primal space, is equivalent to finding common stabbings in the dual space. Concurrency relations for lines correspond to line stabbings [12, 13], while concurrency relations for planes correspond to plane stabbings. When $n$ parameter points, where $n \geq 4$ lie on a stabbing plane in the dual space, the corresponding $n$ planes in the primal space are concurrent. Both conditions are expressed by the same algebraic equation, $P_{\text {conc }}=0$. Furthermore, stabbing planes in the dual space correspond directly to intersection points in 
the primal space. If the stabbing plane has the form $b=\gamma-\alpha a-\beta b$, the common intersection point in the primal space is $(\alpha, \beta, \gamma)$.

For example, for the concurrency relations of Fig. 3, we must find two common stabbings in the dual space, one for the domains $D_{11}, D_{12}, D_{13}, D_{14}$, and a second for $D_{14}, D_{22}, D_{23}, D_{24}$. What complicates the stabbing problem, however, is that the intersection line of the two stabbing planes must cross $D_{14}$. This is not guaranteed by the mere existence of two separate stabbings, which means that the concurrency problem cannot be solved locally.

\section{Constructible Solutions}

Our goal is to show that solutions can be found by geometrical constructions that start from known points. These known points may either be the points used to define the domains, i.e., points in $U$ and $V$, but even when $D$ is given without specifying $U$ and $V$, we can always indicate a set of known points in the primal space.

Definition 1. Let $D$ be a convex, polytopal domain of plane parameters with vertex set $V_{D}$. Let $\left\{\left(a_{i}, b_{i}, c_{i}\right), \ldots\right\}$ be a subset of vertices in $V_{D}$ that lie on a common facet $F$. Then the point $(x, y, z)$ that lies at the intersection of the sets defined by

$$
z=a_{i} x+b_{i} y+c_{i}, \quad \forall\left(a_{i}, b_{i}, c_{i}\right) \in\left(F \cap V_{D}\right)
$$

is called a support point of $D$.

Thus, each facet of the polytope defines a unique support point in the primal space. This support point is defined as the common intersection point for the different positions a plane can take as defined by one facet of its domain. It is also easy to see that the support points of $U$ and $V$ are sufficient to define $D(U, V)$. In fact, if $S$ denotes the set of all support points of $D(U, V)$, then $D(U, V)=D(U \cap S, V \cap S)$.

We will make extensive use of the dualism between the primal and dual space. A vertex $v=(a, b, c)$ in the dual space represents the plane $\Pi$ at the position defined by the equation $z=a x+b y+c$. We will denote this relationship as $v^{\prime}=\Pi$, or vice-versa $\Pi^{\prime}=v$. Similarly, the stabbing plane $\Gamma$ defined as $c=\gamma-a \alpha-b \beta$, with parameters $\alpha, \beta, \gamma$ corresponds to the primal point $p=(\alpha, \beta, \gamma)$. The dual plane $\Gamma$ contains all the parameters of primal planes that pass through the primal point $p$. We will denote this relationship as $p=\Gamma^{\prime}$, or vice-versa $p^{\prime}=\Gamma$. A vertex always lies on at least 3 facets. Hence, a vertex $v$ of a domain is the dual of a plane $v^{\prime}=\Pi$ that contains at least three support points. We will therefore call this primal plane $v^{\prime}$ a support plane. A support plane $v^{\prime}$ is the affine span of a subset of the support points.

\subsection{Meet and Join Operations in the Primal Space}

Suppose we are given a connected chain of $m$ concurrency relations and $n=$ $3 m+1$ domains. Our goal is to show that if the concurrency relations can satisfied 
by $n$ planes, one plane chosen from each domain, then it is always possible to construct geometrically a solution from the support points. From the support points we construct new points and planes by applying either the meet $\wedge$ or the join operator $\vee$. Let $\Pi_{1} \wedge \Pi_{2} \wedge \ldots$ denote the intersection of the planes $\Pi_{1}$, $\Pi_{2}, \ldots$ Let $p_{1} \vee p_{2} \vee \ldots$ denote the affine span of the points $p_{1}, p_{2}, \ldots$ The affine span of three points is a plane, provided the points are in general position. The intersections of two planes is a line, provided the planes are not parallel.

A possible construction for the problem of Fig. 3 may now take the form:

$$
\begin{aligned}
& \Pi_{11}=p_{11} \vee q_{11} \vee s_{11} \\
& \Pi_{12}=p_{12} \vee q_{12} \vee s_{12} \\
& \Pi_{13}=p_{13} \vee q_{13} \vee s_{13} \\
& \Pi_{14}=p_{14} \vee q_{14} \vee\left(\Pi_{11} \wedge \Pi_{12} \wedge \Pi_{13}\right) \\
& \Pi_{22}=p_{22} \vee q_{22} \vee s_{22} \\
& \Pi_{23}=p_{23} \vee q_{23} \vee s_{23} \\
& \Pi_{24}=p_{24} \vee q_{24} \vee\left(\Pi_{14} \wedge \Pi_{22} \wedge \Pi_{23}\right),
\end{aligned}
$$

where $p_{i j}, q_{i j}, s_{i j}$ are support points, and the indices point out the corresponding domain, i.e, $p_{11}$ is a support point of $D_{11}$. However, many more constructions of this form are possible, depending on which support points are selected, and in what order. Our goal is to show that if the concurrency problem has a solution, then there always exists a solution that can be constructed geometrically by meet and join operations on the support points and previously constructed points and planes. Since there are only a finite number of possible constructions, this means that the existence of a solution can be demonstrated or disproved in a finite number of steps.

In a construction like this it is important that all the planes are defined, and that the concurrency relations are automatically satisfied when the points are in general position. For example, the same construction would be invalid if the fourth plane was constructed as $\Pi_{14}=p_{14} \vee q_{14} \vee s_{14}$, because then in general the planes $\Pi_{11}, \Pi_{12}, \Pi_{13}, \Pi_{14}$ would not be concurrent.

\subsection{Meet and Join Operations in the Dual Space}

The meet and join operations of (2) provide one simple example of a possible construction. The constructions that we will need can be much more complicated. In fact, the concurrencies will first be established in the dual space, and then translated into the primal space. In the dual space concurrencies are established by constructing stabbing planes by meet and join operations that start from the vertices of the domains. For example, for the configuration of Fig. 3 we may have a stabbing plane of the form

$$
\Gamma_{1}=v_{11} \vee v_{12} \vee v_{13},
$$

where $v_{i j}$ denotes a vertex of domain $D_{i j}$. But we also allow more complicated expressions of the form

$$
\Gamma_{2}=\left(\Gamma_{1} \wedge\left(v_{14 a} \vee v_{14 b}\right)\right) \vee v_{22} \vee v_{23}
$$


where $\Gamma_{2}$ is constructed from vertices of domains involved in the second relation and a previously constructed stabbing plane $\Gamma_{1}$ from the first relation.

\subsection{Constructible Stabbings Yield Constructible Primal Planes}

Expressions in the dual space can always be translated into expressions that involve support points in the primal space. In fact, each vertex in the dual space corresponds to a support plane in the primal space, which is the affine span of three support points. For example, if the points $p_{1 j}, q_{1 j}, r_{1 j}$ lie on the facet that corresponds to $v_{1 j}$, then

$$
v_{1 j}=\left(p_{1 j} \vee q_{1 j} \vee r_{1 j}\right)^{\prime}
$$

and we have

$$
\Gamma_{1}=\bigvee_{j=1, \ldots, 3}\left(p_{1 j} \vee q_{1 j} \vee r_{1 j}\right)^{\prime},
$$

which specifies how the stabbing plane can be constructed by taking duals from affine spans of support points.

However, the construction of the stabbing planes in terms of support points still leaves some freedom with respect to the parameters of the primal planes $\Pi_{i j}$. Suppose that we have constructed $m$ stabbing planes from the domain vertices such that the intersection line $\Gamma_{n} \wedge \Gamma_{n+1}$ crosses the domain $D_{n 4}$ for each subsequent pair. Then we still have to make clear how we can derive the parameters of the planes $\Pi_{i j}$ that satisfy the concurrency relations. To this end we will select in each domain a parameter point that lies on the stabbing plane, but where necessary we introduce additional constraints, which are explained below, to remove remaining degrees of freedom.

The additional constraints are chosen as simple as possible, e.g., by requiring that a parameter point lies on a edge of a domain. We have to make a distinction between domains that are involved either in one or in two relations. First, for a domain $D_{i j}$ involved in one relation, if the stabbing plane $\Gamma_{i}$ passes through one or more vertices of $D_{i j}$, we select one of these vertices. If $\Gamma_{i}$ contains no vertex, the stabbing plane still passes through at least one edge of domain $D_{i j}$. In this case we select the intersection point of the edge and the stabbing plane. Second, for a domain $D_{i 4}$ that is involved in two relations, we select the intersection point of a facet of $D_{i 4}$ and the intersection line $\Gamma_{i} \wedge \Gamma_{i+1}$. Clearly, all the selected parameter points are constructible in the dual space, since they lie either at a vertex, at the intersection of an edge and a constructible stabbing plane, or at the intersection of a facet and two constructible stabbing planes. For example, $\left(\Gamma_{1} \wedge \Gamma_{2} \wedge\left(u_{14} \vee v_{14} \vee w_{14}\right)\right)^{\prime}$ represents a plane in the primal space whose parameter point is defined in the dual space as the intersection of two stabbing planes and a facet spanned by the vertices $u_{14}, v_{14}, w_{14}$. In turn, each vertex can be written as the dual of the affine span of three support points, e.g., $u_{14}=\left(p_{14} \vee q_{14} \vee s_{14}\right)^{\prime}$. Hence, when a parameter point $(a, b, c)$ is constructible in the dual space from the vertices, the corresponding plane $\Pi_{(a, b, c)}$ is constructible in the primal space from the support points. 


\subsection{Proof of the Main Result}

We start with a simple result for one concurrency relation with 4 primal planes.

Proposition 1. If four domains can be stabbed by a common dual plane, then there is a dual plane passing through 3 domain vertices that stabs the fourth domain.

Proof. The requested stabbing is easily found by three rotations. Let $a_{11}, a_{12}, a_{13}, a_{14}$ denote four arbitrarily chosen points on the common stabbing plane, one point chosen from each domain, i.e., $a_{i j} \in D_{i j}$. We let the stabbing plane rotate around the axis $a_{13} a_{14}$ until we hit a vertex in one of the two other domains. Without loss of generality we assume that this vertex lies in $D_{11}$ and call it $v_{11}$. Next we let the plane rotate around the axis $a_{14} v_{11}$ until we hit a second vertex either in $D_{12}$ or $D_{13}$. Without loss of generality we may assume this vertex lies in $D_{12}$, and call it $v_{12}$. Finally, we let the plane rotate around $v_{11} v_{12}$, until we hit a third vertex either in $D_{13}$ or $D_{14}$. The resulting plane passes through 3 vertices in 3 different domains and still stabs the fourth domain.

Note that a domain vertex corresponds to a supporting plane. Thus the above result translates into: there exist three supporting planes, such that there is a fourth separating plane that passes through the common intersection point of the three supporting planes and two support points of the fourth domain.

To prove the main theorem, we will extend the previous result. However, in order to ensure that the stabbings stay within the common domains $D_{i 4}$, we introduce visual hulls in the dual, which should not be confused with the visual hulls of Section 11. Suppose we have a convex polytope $D$, and two vertices $v_{1}$ and $v_{2}$ not in $D$. We define a visual hull of $D$ as seen from the line $v_{1} \vee v_{2}$ as follows:

$$
H\left(v_{1} \vee v_{2}, D\right)=\left\{p \in \mathbb{R}^{3}:\left(p \vee v_{1} \vee v_{2}\right) \cap D \neq \emptyset\right\}
$$

In other words the visual hull is the set of all points that lie on planes that pass through $v_{1} \vee v_{2}$ and cross $D$. Note that this a variant of the more common definition of a visual hull. In our case the light rays are not lines but planes, and the projection center is not a point but a line. It is also clear that the intersection of a visual hull $H\left(v_{1} \vee v_{2}, D\right)$ with another domain $D^{\prime}$ is again a polytope, whose vertices can be constructed by meet and join operations on $v_{1}, v_{2}$, the vertices of $D$, and the vertices of $D^{\prime}$.

We will now prove the general theorem. The proof is given in the dual space, but it is helpful to keep the dualities of Table1 in mind. The dual space column in Table11indicates how the primal plane can be constructed from domain vertices and duals of stabbing planes.

Theorem 1. Suppose we have a connected chain of $m$ concurrency relations, with $3 n+1$ domains $D_{11}, \ldots, D_{m 4}$. If there exist $3 n+1$ primal planes $\Phi_{11}, \ldots, \Phi_{m 4}$, with $\Phi_{i j}^{\prime} \in D_{i j}$, that satisfy the concurrency relations, then there is a second set of primal planes $\Pi_{11}, \ldots, \Pi_{m 4}$ with $\Pi_{i j}^{\prime} \in D_{i j}$, where each $\Pi_{i j}$ can be constructed by meet and join operations on the support points and previously constructed planes. 
Table 1. Dualities between primal and dual space

\begin{tabular}{l|l} 
dual space & primal space \\
\hline$\Gamma_{i}$ passes through vertex $v$ & support plane spanned by 3 support points of $v^{\prime}$ \\
$\Gamma_{i}$ crosses edge $v_{1} v_{2}$ & plane spanned by 2 support points in $v_{1}^{\prime} \cap v_{2}^{\prime}$ and by $\Gamma_{i}^{\prime}$ \\
line $\Gamma_{i} \wedge \Gamma_{i+1}$ crosses facet & plane spanned by 1 support point, $\Gamma_{i}^{\prime}$ and $\Gamma_{i+1}^{\prime}$ \\
\hline
\end{tabular}

Proof. The general idea of the proof is to start from stabbing planes in the dual space that correspond to a solution, which is known to exist, and then rotate the stabbing planes until they pass through a sufficient number of vertices, edges or facets of the domains.

First Stage. We start with the first relation in the chain. Since there is a solution, there is also stabbing plane $\Gamma_{1}$ in the dual space that stabs the 4 domains of the first relation. Let $a_{11}, a_{12}, a_{13}$ denote three arbitrarily chosen points on the common stabbing plane, one point chosen from each domain $D_{11}, D_{12}, D_{13}$. Let $a_{14}$ be a point chosen from $D_{14}$ on the line $\Gamma_{1} \cap \Gamma_{2}$. We let the stabbing plane $\Gamma_{1}$ rotate around the axis $a_{13} \vee a_{14}$ until we hit a vertex in one of the two other domains $D_{11}$ or $D_{12}$. Without loss of generality we assume that this is a vertex of $D_{11}$, which we call $v_{11}$. Next we let the plane rotate around the axis $a_{14} \vee v_{11}$, until we hit a second vertex either in $D_{12}$ or $D_{13}$. Assume this vertex lies in $D_{12}$, and call it $v_{12}$.

We now have a stabbing plane $\Gamma_{1}$ that passes through the vertices $v_{11} \in D_{11}$ and $v_{12} \in D_{12}$. Furthermore, $a_{14}$ still lies on the rotated stabbing plane, as well as on $\Gamma_{2}$. In principle, it is possible to let $\Gamma_{1}$ rotate further around the axis $v_{11} \vee v_{12}$ until it hits a third vertex in either $D_{13}$ or $D_{14}$, which would then completely determine the position of $\Gamma_{1}$. However, there is a risk that the resulting plane would cross $D_{14}$ in a part that can no longer be reached by $\Gamma_{2}$. We will therefore proceed with $\Gamma_{2}$. But also when rotating $\Gamma_{2}$ we run the risk that it will cross $D_{14}$ in a part that is no longer reachable by $\Gamma_{1}$. To avoid this, we introduce the visual hull of $D_{13}$ as seen from the axis $v_{11} \vee v_{12}$, and we replace $D_{14}$ by

$$
\hat{D}_{14}=D_{14} \cap H\left(v_{11} \vee v_{12}, D_{13}\right) \text {. }
$$

Clearly, for any point $a_{14}$ in $\hat{D}_{14}$, we can always find a plane that passes through the axis $v_{11} \vee v_{12}$ and that crosses $D_{13}$, since in (3) the visual hull was defined in a way to guarantee this. As mentioned previously, the visual hull can be constructed by joins and meets of domain vertices.

To proceed we choose arbitrary points $a_{23} \in D_{23}$ and $a_{24} \in D_{24}$ and let $\Gamma_{2}$ rotate around the axis $a_{23} \vee a_{24}$ until we hit a vertex in $\hat{D}_{14}$ or $D_{22}$, for example, $v_{22} \in D_{22}$. Next, we let the plane rotate around the axis $v_{22} \wedge a_{24}$ until we hit a second vertex, for example, the vertex $v_{14}$ of $\hat{D}_{14}$. Note that this vertex may or may not be a vertex of the original domain $D_{14}$, but it is always constructible.

As soon as $\Gamma_{2}$ passes through two vertices, we compute the visual hull of the third domain, chosen such that it is not equal to $D_{24}$, and as seen from the axis 
passing through the two vertices already selected. For example, if the vertices are $v_{22}$ and $v_{14}$, we compute

$$
\hat{D}_{24}=D_{24} \cap H\left(v_{14} \vee v_{22}, D_{23}\right)
$$

and replace $D_{24}$ by $\hat{D}_{24}$. The previous step is now repeated until we have found $m$ stabbing planes that contain two vertices each.

Second Stage. We note that for the last stabbing plane $\Gamma_{m}$, it is no longer a problem to rotate it around its two vertices, since the last domain in the configuration, $D_{m 4}$, is not used in any $(m+1)$-th concurrency relation. Hence, by rotating the last plane around the axis passing through the two vertices that have already been determined, we can determine a third vertex for the last plane. As soon as this vertex is known, we can go through the configuration in the opposite direction and determine a third vertex for each of the stabbing planes.

While doing this we have to replace the domains $D_{i 4}$ by their intersection with a stabbing plane. Suppose, for example, that there are three relations, as in Fig. 4 and that we have found vertices $v_{32}, v_{33}, v_{34}$ that completely determine the third stabbing plane $\Gamma_{3}=v_{32} \vee v_{33} \vee v_{34}$. To ensure that the second stabbing plane $\Gamma_{2}$ will be able to meet $\Gamma_{3}$ the third stabbing plane within the domain $\hat{D}_{24}$, we replace $\hat{D}_{24}$ by

$$
\tilde{D}_{24}=\hat{D}_{24} \cap\left(v_{32} \vee v_{33} \vee v_{34}\right) .
$$

Note that $\tilde{D}_{24}$ is a planar polygon. Next we rotate the second last plane until it hits a third vertex, and replace the polytopes $\hat{D}_{i 4}$ when necessary by polygons

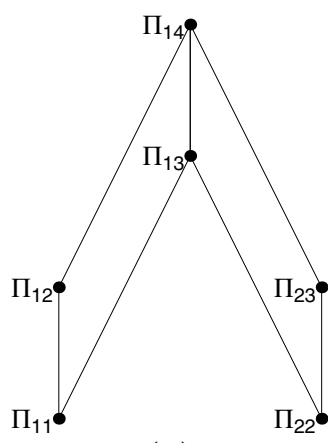

(a)

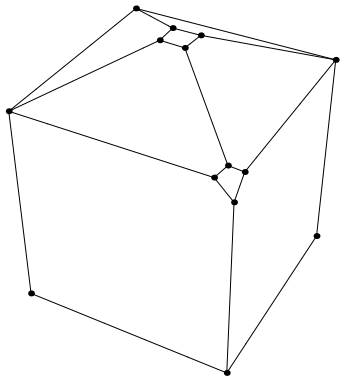

(b)

Fig. 5. Theorem [1 is not valid for the chain shown in (a) where 2 relations share more than one plane. (b) shows how this chain can arise in primal space. A polytope has two small facets, each adjacent to 4 larger facets. By changing the position of the large facets slightly, the small facets could disappear and be replaced by two vertices. However, two of the large facets are adjacent to both smaller facets. Each of the six large facets in (b) corresponds to a plane $\Pi_{i j}$ in diagram (a). The planes $\Pi_{13}$ and $\Pi_{14}$ correspond to the large facets incident to both small facets. 
$\tilde{D}_{i 4}$, until all the stabbing planes pass through constructible vertices in the dual space. Note that these vertices may either be vertices of the original domains, vertices of intersections of domains and visual hulls, or vertices of the polygons that result from taking the intersection of a domain, a visual hull and a stabbing plane already found.

After all the stabbing planes have been constructed, constructible primal planes can be pinned down as explained in Section 3.3.

\section{Conclusion}

We have shown that if a solution of the concurrency problem exists, it can be constructed in a finite number of steps. We did not specify how many steps are necessary. Such more detailed analysis has been performed for line configurations in [10], but not yet for planes. Furthermore, the main result is only valid for connected chains where two concurrency relations share only one plane. In fact, when relations share two or more planes, as in Fig. [5, one can easily find examples where a solution cannot be constructed in a simple way from the support points. It remains to examine whether geometric constructions are possible for more complicated configurations than the ones studied here.

\section{References}

1. He, P., Edalat, A.: Visual hull from imprecise polyhedral scene. In: Goesele, M., Matsushita, Y., Sagawa, R., Yang, R. (eds.) 3DIMPVT, pp. 164-171. IEEE (2011)

2. Laurentini, A.: The visual hull concept for silhouette-based image understanding. IEEE Trans. Pattern Anal. Mach. Intell. 16(2), 150-162 (1994)

3. Petitjean, S.: A computational geometric approach to visual hulls. Int. J. Comput. Geometry Appl. 8(4), 407-436 (1998)

4. Labatut, P., Pons, J.P., Keriven, R.: Efficient multi-view reconstruction of largescale scenes using interest points, delaunay triangulation and graph cuts. In: ICCV, pp. 1-8. IEEE (2007)

5. Kim, H., Sakamoto, R., Kitahara, I., Toriyama, T., Kogure, K.: Robust Foreground Extraction Technique Using Gaussian Family Model and Multiple Thresholds. In: Yagi, Y., Kang, S.B., Kweon, I.S., Zha, H. (eds.) ACCV 2007, Part I. LNCS, vol. 4843, pp. 758-768. Springer, Heidelberg (2007)

6. Zivkovic, Z., van der Heijden, F.: Efficient adaptive density estimation per image pixel for the task of background subtraction. Pattern Recognition Letters 27(7), $773-780(2006)$

7. Kim, C.E.: Three-dimensional digital planes. IEEE Trans. Pattern Anal. Machine Intell. 6, 639-645 (1984)

8. Andres, E., Acharya, R., Sibata, C.: Discrete analytical hyperplanes. Graphical Models and Image Processing 59(5), 302-309 (1997)

9. Jamet, D., Toutant, J.L.: Minimal arithmetic thickness connecting discrete planes. Discrete Applied Mathematics 157(3), 500-509 (2009)

10. Veelaert, P., Heyvaert, M.: Reconstruction of Concurrent Lines from Leaning Points. In: Aggarwal, J.K., Barneva, R.P., Brimkov, V.E., Koroutchev, K.N., Korutcheva, E.R. (eds.) IWCIA 2011. LNCS, vol. 6636, pp. 182-193. Springer, Heidelberg (2011) 
11. Coeurjolly, D., Sivignon, I., Dupont, F., Feschet, F., Chassery, J.M.: On digital plane preimage structure. Discrete Applied Mathematics 151(1-3), 78-92 (2005)

12. Veelaert, P.: Algorithms that measure parallelism and concurrency of lines in digital images. In: Proc. of SPIE's Conference on Vision Geometry VIII, Denver. SPIE, pp. 69-79 (1999)

13. Veelaert, P.: Concurrency of Line Segments in Uncertain Geometry. In: Braquelaire, A., Lachaud, J.-O., Vialard, A. (eds.) DGCI 2002. LNCS, vol. 2301, pp. 289-300. Springer, Heidelberg (2002) 